Article

\title{
New Conformable Fractional Integral Inequalities of Hermite-Hadamard Type for Convex Functions
}

\author{
Pshtiwan Othman Mohammed * ${ }^{\mathbb{D}}$ and Faraidun Kadir Hamasalh \\ Department of Mathematics, College of Education, University of Sulaimani, Sulaimani 46001, Kurdistan Region, \\ Iraq; faraidunsalh@gmail.com \\ * Correspondence: pshtiwansangawi@gmail.com
}

Received: 28 December 2018; Accepted: 8 February 2019; Published: 20 February 2019

check for updates

Abstract: In this work, we established new inequalities of Hermite-Hadamard type for convex functions via conformable fractional integrals. Through the conformable fractional integral inequalities, we found some new inequalities of Hermite-Hadamard type for convex functions in the form of classical integrals.

Keywords: convex function; integral inequalities; Hermite-Hadamard inequality; conformable fractional integrals

\section{Introduction}

A function $h: \mathcal{I} \subseteq \mathbb{R} \rightarrow \mathbb{R}$ is said to be convex on the interval $\mathcal{I}$, if the inequality

$$
h(\zeta x+(1-\zeta) y) \leq \zeta h(x)+(1-\zeta) h(y)
$$

holds for all $x, y \in \mathcal{I}$ and $\zeta \in[0,1]$. We say that $h$ is concave if $-h$ is convex.

For convex functions, many equalities or inequalities have been established by many authors; for example the Ostrowski type inequality [1], Hardy type inequality [2], Olsen type inequality [3], and Gagliardo-Nirenberg type inequality [4] but the most common and significant inequality is the Hermite-Hadamard type inequality [5,6], which is defined as:

$$
h\left(\frac{u+v}{2}\right) \leq \frac{1}{v-u} \int_{u}^{v} h(x) d x \leq \frac{h(u)+h(v)}{2},
$$

where the function $h: \mathcal{I} \subseteq \mathbb{R} \rightarrow \mathbb{R}$ is assumed to be a convex function with $u<v$ and $u, v \in \mathcal{I}$.

A number of mathematicians in the field of applied and pure mathematics have dedicated their efforts to extend, generalize, counterpart, and refine the Hermite-Hadamard inequality (2) for different classes of convex functions and mappings. For more recent results obtained on inequality (2), we refer the reader to References [5,7-10].

Definition 1 ([11]). Suppose that $h \in L([u, v])$. The left and right Riemann-Liouville fractional integrals $J_{u^{+}}^{\alpha} h$ and $J_{v^{-}}^{\alpha} h$ of order $\alpha>0$ are defined by

$$
J_{u^{+}}^{\alpha} h(x)=\frac{1}{\Gamma(\alpha)} \int_{u}^{x}(x-\zeta)^{\alpha-1} h(\zeta) d \zeta, \quad x>u,
$$


and

$$
J_{v^{-}}^{\alpha} h(x)=\frac{1}{\Gamma(\alpha)} \int_{x}^{v}(\zeta-x)^{\alpha-1} h(\zeta) d \zeta, \quad x<v,
$$

respectively, where $\Gamma(\alpha)$ is the standard gamma function defined by $\Gamma(\alpha)=\int_{0}^{\infty} e^{-\zeta} \zeta^{\alpha-1} d \zeta$ and $J_{u^{+}}^{0} h(x)=$ $J_{v^{-}}^{0} h(x)=h(x)$.

As for classical integrals, many Hermite-Hadamard type inequalities have been established for the Riemann-Liouville fractional integrals; for more details and interesting applications see References $[6,12,13]$.

Now, we give the definition of the conformable fractional derivative with its important properties which are useful in order to obtain our main results (see References [14-21]). In our study, we use the Katugampola derivative formulation of conformable derivative which is explained in the following definition:

Definition 2 ([20]). Given a function $h:[0, \infty) \rightarrow \mathbb{R}$. Then, the conformable fractional derivative of $h$ of order $\alpha$ of $h$ at $\zeta$ is defined by

$$
D_{\alpha}(h)(\zeta)=\lim _{\epsilon \rightarrow 0} \frac{h\left(\zeta+\epsilon \zeta^{1-\alpha}\right)-h(\zeta)}{\epsilon}, \quad \alpha \in(0,1), \zeta>0 .
$$

If $h$ is $\alpha$-differentiable in some $(0, \alpha), \alpha>0, \lim _{\zeta \rightarrow 0^{+}} h^{(\alpha)}(\zeta)$ exist, then define

$$
h^{(\alpha)}(0)=\lim _{\zeta \rightarrow 0^{+}} h^{(\alpha)}(\zeta) .
$$

Additionally, note that if $h$ is differentiable, then

$$
D_{\alpha}(h)(\zeta)=\zeta^{1-\alpha} h^{\prime}(\zeta), \quad \text { where } h^{\prime}(\zeta)=\lim _{\epsilon \rightarrow 0} \frac{h(\zeta+\epsilon)-h(\zeta)}{\epsilon} .
$$

We can write $h^{(\alpha)}(\zeta)$ for $D_{\alpha}(h)(\zeta)$ or $\frac{d_{\alpha}}{d_{\alpha} \zeta}(h(\zeta))$ to denote the conformable fractional derivatives of $h$ of order $\alpha$ at $\zeta$. In addition, if the conformable fractional derivative of $h$ of order $\alpha$ exists, then we simply say $h$ is $\alpha$-differentiable.

Theorem 1 ([20]). Let $\alpha \in(0,1]$ and $h, g$ be $\alpha$-differentiable at a point $\zeta>0$. Then,

1. $D_{\alpha}(u f+v g)=u D_{\alpha}(h)+v D_{\alpha}(g)$ for all $u, v \in \mathbb{R}$,

2. $D_{\alpha}(h g)=h D_{\alpha}(g)+g D_{\alpha}(h)$,

3. $D_{\alpha}\left(\frac{h}{g}\right)=\frac{h D_{\alpha}(g)-g D_{\alpha}(h)}{g^{2}}$,

4. $D_{\alpha}(c)=0$ for all constant function $h(\zeta)=c$,

5. $D_{\alpha}(1)=0$,

6. $D_{\alpha}\left(\frac{1}{\alpha} \zeta^{\alpha}\right)=1$.

Now, we give the definition of conformable fractional integral:

Definition 3 ([16]). Let $\alpha \in(0,1]$ and $0 \leq u \leq v$. We say that a function $h:[u, v] \rightarrow \mathbb{R}$ is $\alpha$-fractional integrable on $[u, v]$, if the integral

$$
\int_{u}^{v} h(\zeta) d_{\alpha} \zeta=\int_{u}^{v} h(\zeta) \zeta^{\alpha-1} d \zeta
$$


exists and is finite.

Remark 1. (u) All $\alpha$-fractional integrable functions on $[u, v]$ are indicated by $L_{\alpha}^{1}([u, v])$.

(v) For the usual Riemann improper integral and $\alpha \in(0,1]$, we have

$$
I_{\alpha}^{u}(h)(\zeta)=I_{1}^{a}\left(\zeta^{\alpha-1} h\right)=\int_{u}^{\zeta} x^{\alpha-1} h(x) d x .
$$

The aim of our article is to establish some new inequalities connected with the Hermite-Hadamard inequalities (2) via conformable fractional integral.

\section{Main Results}

Our main results depend on the following equality:

Lemma 1. Let $h:[u, v] \subseteq \mathbb{R} \rightarrow \mathbb{R}$ be an $\alpha$-fractional differentiable mapping on $(u, v)$ with $0 \leq u<v$. If $D_{\alpha}(h) \in L_{\alpha}^{1}([u, v])$, then the following identity for conformable fractional integral holds:

$$
\Phi_{\alpha}(u, v)=\sum_{i=1}^{4} \delta_{i}
$$

where

$$
\begin{aligned}
\delta_{1} & =\frac{v-u}{4} \int_{0}^{1}\left[\left(u \zeta+(1-\zeta) \frac{3 u+v}{4}\right)^{2 \alpha-1}-\left(\frac{3 u+v}{4}\right)^{\alpha}\left(u \zeta+(1-\zeta) \frac{3 u+v}{4}\right)^{\alpha-1}\right] \\
& \times D_{\alpha}(h)\left(u \zeta+(1-\zeta) \frac{3 u+v}{4}\right) d_{\alpha} \zeta, \\
\delta_{2} & =\frac{v-u}{4} \int_{0}^{1}\left[\left(\frac{3 u+v}{4} \zeta+(1-\zeta) \frac{u+v}{2}\right)^{2 \alpha-1}-\left(\frac{3 u+v}{4}\right)^{\alpha}\left(\frac{3 u+v}{4} \zeta+(1-\zeta) \frac{u+v}{2}\right)^{\alpha-1}\right] \\
& \times D_{\alpha}(h)\left(\frac{3 u+v}{4} \zeta+(1-\zeta) \frac{u+v}{2}\right) d_{\alpha} \zeta, \\
\delta_{3} & =\frac{v-u}{4} \int_{0}^{1}\left[\left(\frac{u+v}{2} \zeta+(1-\zeta) \frac{u+3 v}{4}\right)^{2 \alpha-1}-\left(\frac{u+3 v}{4}\right)^{\alpha}\left(\frac{u+v}{2} \zeta+(1-\zeta) \frac{u+3 v}{4}\right)^{\alpha-1}\right] \\
& \times D_{\alpha}(h)\left(\frac{u+v}{2} \zeta+(1-\zeta) \frac{u+3 v}{4}\right) d_{\alpha} \zeta, \\
\delta_{4} & =\frac{v-u}{4} \int_{0}^{1}\left[\left(\frac{u+3 v}{4} \zeta+(1-\zeta) v\right)^{2 \alpha-1}-\left(\frac{u+3 v}{4}\right)^{\alpha}\left(\frac{u+3 v}{4} \zeta+(1-\zeta) v\right)^{\alpha-1}\right] \\
& \times D_{\alpha}(h)\left(\frac{u+3 v}{4} \zeta+(1-\zeta) v\right) d_{\alpha} \zeta,
\end{aligned}
$$

and

$$
\begin{aligned}
\Phi_{\alpha}(u, v) & =\left[\left(\frac{3 u+v}{4}\right)^{\alpha}-u^{\alpha}\right] h(u)+\left[v^{\alpha}-\left(\frac{u+3 v}{4}\right)^{\alpha}\right] h(v) \\
& +\left[\left(\frac{u+3 v}{4}\right)^{\alpha}-\left(\frac{3 u+v}{4}\right)^{\alpha}\right] h\left(\frac{u+v}{2}\right)-\alpha \int_{u}^{v} h(x) d_{\alpha} x .
\end{aligned}
$$


Proof. By using the definition of the conformable fractional derivative (4), we have

$$
\delta_{1}=\frac{v-u}{4} \int_{0}^{1}\left[\left(u \zeta+(1-\zeta) \frac{3 u+v}{4}\right)^{\alpha}-\left(\frac{3 u+v}{4}\right)^{\alpha}\right] h^{\prime}\left(u \zeta+(1-\zeta) \frac{3 u+v}{4}\right) d \zeta .
$$

On integrating by parts, one can have

$$
\delta_{1}=\left[\left(\frac{3 u+v}{4}\right)^{\alpha}-u^{\alpha}\right] h(u)-\alpha \int_{0}^{1}\left(u \zeta+(1-\zeta) \frac{3 u+v}{4}\right)^{\alpha-1} h\left(u \zeta+(1-\zeta) \frac{3 u+v}{4}\right) d \zeta
$$

Using the change of the variable $x:=u \zeta+(1-\zeta) \frac{3 u+v}{4}, \zeta \in[0,1]$ and definition of conformable fractional integral (5), we obtain

$$
\begin{aligned}
\delta_{1} & =\left[\left(\frac{3 u+v}{4}\right)^{\alpha}-u^{\alpha}\right] h(u)-\alpha \int_{u}^{\frac{3 u+v}{4}} x^{\alpha-1} h(x) d x \\
& =\left[\left(\frac{3 u+v}{4}\right)^{\alpha}-u^{\alpha}\right] h(u)-\alpha \int_{u}^{\frac{3 u+v}{4}} h(x) d_{\alpha} x .
\end{aligned}
$$

Similarly, we get

$$
\begin{aligned}
& \delta_{2}=\left[\left(\frac{u+v}{2}\right)^{\alpha}-\left(\frac{3 u+v}{4}\right)^{\alpha}\right] h\left(\frac{u+v}{2}\right)-\alpha \int_{\frac{3 u+v}{4}}^{\frac{u+v}{2}} h(x) d_{\alpha} x \\
& \delta_{3}=\left[\left(\frac{u+3 v}{4}\right)^{\alpha}-\left(\frac{u+v}{2}\right)^{\alpha}\right] h\left(\frac{u+v}{2}\right)-\alpha \int_{\frac{u+v}{2}}^{\frac{u+3 v}{4}} h(x) d_{\alpha} x,
\end{aligned}
$$

and

$$
\delta_{4}=\left[v^{\alpha}-\left(\frac{u+3 v}{4}\right)^{\alpha}\right] h(v)-\alpha \int_{\frac{u+3 v}{4}}^{v} h(x) d_{\alpha} x .
$$

Adding $\delta_{1}, \delta_{2}, \delta_{3}$ and $\delta_{4}$ together, we obtain the desired identity (7). This completes the proof of Lemma 1.

Remark 2. With the similar assumptions of Lemma 1, if $\alpha=1$, then identity (7) reduces to the following identity:

$$
\begin{aligned}
& \Phi_{1}(u, v)=\int_{0}^{1}(1-\zeta) h^{\prime}\left(\frac{3 u+v}{4} \zeta+(1-\zeta) \frac{u+v}{2}\right) d \zeta-\int_{0}^{1} t h^{\prime}\left(u \zeta+(1-\zeta) \frac{3 u+v}{4}\right) d \zeta \\
& +\int_{0}^{1}(1-\zeta) h^{\prime}\left(\frac{u+3 v}{4} \zeta+(1-\zeta) v\right) d \zeta-\int_{0}^{1} t h^{\prime}\left(\frac{u+v}{2} \zeta+(1-\zeta) \frac{u+3 v}{4}\right) d \zeta,
\end{aligned}
$$

where

$$
\Phi_{1}(u, v)=\frac{1}{2}\left[\frac{h(u)+h(v)}{2}+h\left(\frac{u+v}{2}\right)\right]-\frac{4}{v-u} \int_{u}^{v} h(x) d x
$$

which is obtained by Shi et al. [12]. 
Theorem 2. Let $h:[u, v] \subseteq \mathbb{R} \rightarrow \mathbb{R}$ be an $\alpha$-fractional differentiable mapping on $(u, v)$ with $0 \leq u<v$. If $D_{\alpha}(h) \in L_{\alpha}^{1}([u, v])$ and $\left|h^{\prime}\right|$ is convex on $[u, v]$, then the following inequality for conformable fractional integral holds:

$$
\begin{aligned}
\left|\Phi_{\alpha}(u, v)\right| & \leq \frac{v-u}{4}\left[d_{1}(\alpha)\left|h^{\prime}(u)\right|+d_{2}(\alpha)\left|h^{\prime}(v)\right|\right. \\
& \left.+d_{3}(\alpha)\left|h^{\prime}\left(\frac{3 u+v}{4}\right)\right|+d_{4}(\alpha)\left|h^{\prime}\left(\frac{u+v}{2}\right)\right|+d_{5}(\alpha)\left|h^{\prime}\left(\frac{u+3 v}{4}\right)\right|\right],
\end{aligned}
$$

where

$$
\begin{gathered}
d_{1}(\alpha)=\frac{1}{12}\left[4 u^{\alpha}+\left(\frac{3 u+v}{4}\right) u^{\alpha-1}+\left(\frac{3 u+v}{4}\right)^{\alpha-1} u-5\left(\frac{3 u+v}{4}\right)^{\alpha}\right], \\
d_{2}(\alpha)=\frac{1}{12}\left[3 v^{\alpha}+\left(\frac{3 u+v}{4}\right) v^{\alpha-1}+\left(\frac{3 u+v}{4}\right)^{\alpha-1} v-5\left(\frac{3 u+v}{4}\right)^{\alpha}\right], \\
d_{3}(\alpha)=\frac{1}{12}\left[u^{\alpha}+\left(\frac{3 u+v}{4}\right) u^{\alpha-1}+\left(\frac{3 u+v}{4}\right)^{\alpha-1} u+\left(\frac{u+v}{2}\right)\left(\frac{3 u+v}{4}\right)^{\alpha-1}\right. \\
\left.+\left(\frac{u+v}{2}\right)^{\alpha-1}\left(\frac{3 u+v}{4}\right)+\left(\frac{u+v}{2}\right)^{\alpha}-5\left(\frac{3 u+v}{4}\right)^{\alpha}\right], \\
d_{4}(\alpha)=\frac{1}{12}\left[7\left(\frac{u+v}{2}\right)^{\alpha}+\left(\frac{u+v}{2}\right)\left(\frac{3 u+v}{4}\right)^{\alpha-1}+\left(\frac{u+v}{2}\right)^{\alpha-1}\left(\frac{3 u+v}{4}\right)\right. \\
\left.+\left(\frac{u+v}{2}\right)\left(\frac{u+3 v}{4}\right)^{\alpha-1}+\left(\frac{u+v}{2}\right)^{\alpha-1}\left(\frac{u+3 v}{4}\right)-5\left(\frac{3 u+v}{4}\right)^{\alpha}-5\left(\frac{u+3 v}{4}\right)^{\alpha}\right], \\
d_{5}(\alpha)=\frac{1}{12}\left[v^{\alpha}+\left(\frac{u+v}{2}\right)^{\alpha}+\left(\frac{u+3 v}{4}\right)\left(\frac{u+v}{2}\right)^{\alpha-1}+\left(\frac{u+3 v}{4}\right)^{\alpha-1}\left(\frac{u+v}{2}\right)\right. \\
\left.+v\left(\frac{u+3 v}{4}\right)^{\alpha-1}+v^{\alpha-1}\left(\frac{u+3 v}{4}\right)-5\left(\frac{u+3 v}{4}\right)^{\alpha}\right] .
\end{gathered}
$$

Proof. Using Lemma 1 and the property (4), we have

$$
\begin{aligned}
& \Phi_{\alpha}(u, v)=\frac{v-u}{4}\left\{\int_{0}^{1}\left[\left(u \zeta+(1-\zeta) \frac{3 u+v}{4}\right)^{\alpha}-\left(\frac{3 u+v}{4}\right)^{\alpha}\right] h^{\prime}\left(u \zeta+(1-\zeta) \frac{3 u+v}{4}\right) d \zeta\right. \\
& +\int_{0}^{1}\left[\left(\frac{3 u+v}{4} \zeta+(1-\zeta) \frac{u+v}{2}\right)^{\alpha}-\left(\frac{3 u+v}{4}\right)^{\alpha}\right] h^{\prime}\left(\frac{3 u+v}{4} \zeta+(1-\zeta) \frac{u+v}{2}\right) d \zeta \\
& +\int_{0}^{1}\left[\left(\frac{u+v}{2} \zeta+(1-\zeta) \frac{u+3 v}{4}\right)^{\alpha}-\left(\frac{u+3 v}{4}\right)^{\alpha}\right] h^{\prime}\left(\frac{u+v}{2} \zeta+(1-\zeta) \frac{u+3 v}{4}\right) d_{\alpha} \zeta \\
& \left.+\int_{0}^{1}\left[\left(\frac{u+3 v}{4} \zeta+(1-\zeta) v\right)^{\alpha}-\left(\frac{u+3 v}{4}\right)^{\alpha}\right] h^{\prime}\left(\frac{u+3 v}{4} \zeta+(1-\zeta) v\right) d_{\alpha} \zeta\right\} .
\end{aligned}
$$


By using the convexity of $x^{\alpha-1}$ for $x>0, \alpha \in(0,1]$, we have

$$
\begin{aligned}
& \left(u \zeta+(1-\zeta) \frac{3 u+v}{4}\right)^{\alpha}-\left(\frac{3 u+v}{4}\right)^{\alpha}=\left(u \zeta+(1-\zeta) \frac{3 u+v}{4}\right)^{\alpha-1+1}-\left(\frac{3 u+v}{4}\right)^{\alpha} \\
& =\left(u \zeta+(1-\zeta) \frac{3 u+v}{4}\right)^{\alpha-1}\left(u \zeta+(1-\zeta) \frac{3 u+v}{4}\right)-\left(\frac{3 u+v}{4}\right)^{\alpha} \\
& \leq\left[u^{\alpha-1} \zeta+(1-\zeta)\left(\frac{3 u+v}{4}\right)^{\alpha-1}\right]\left(u \zeta+(1-\zeta) \frac{3 u+v}{4}\right)-\left(\frac{3 u+v}{4}\right)^{\alpha}, \\
& \left(\frac{3 u+v}{4} \zeta+(1-\zeta) \frac{u+v}{2}\right)^{\alpha}-\left(\frac{3 u+v}{4}\right)^{\alpha} \\
& \leq\left[t\left(\frac{3 u+v}{4}\right)^{\alpha-1}+(1-\zeta)\left(\frac{u+v}{2}\right)^{\alpha-1}\right]\left(\frac{3 u+v}{4} \zeta+(1-\zeta) \frac{u+v}{2}\right)-\left(\frac{3 u+v}{4}\right)^{\alpha}, \\
& \left(\frac{u+v}{2} \zeta+(1-\zeta) \frac{u+3 v}{2}\right)^{\alpha}-\left(\frac{u+3 v}{4}\right)^{\alpha} \\
& \leq\left[t\left(\frac{u+v}{2}\right)^{\alpha-1}+(1-\zeta)\left(\frac{u+3 v}{4}\right)^{\alpha-1}\right]\left(\frac{u+v}{2} \zeta+(1-\zeta) \frac{u+3 v}{4}\right)-\left(\frac{u+3 v}{4}\right)^{\alpha},
\end{aligned}
$$

and

$$
\begin{aligned}
& \left(\frac{u+3 v}{4} \zeta+(1-\zeta) v\right)^{\alpha}-\left(\frac{u+3 v}{4}\right)^{\alpha} \\
& \leq\left[t\left(\frac{u+3 v}{4}\right)^{\alpha-1}+(1-\zeta) v^{\alpha-1}\right]\left(\frac{u+3 v}{4} \zeta+(1-\zeta) v\right)-\left(\frac{u+3 v}{4}\right)^{\alpha} .
\end{aligned}
$$

Using (11), (12), (13), and (14) in (10) and using the properties of modulus, we get

$$
\begin{aligned}
& \left|\Phi_{\alpha}(u, v)\right| \leq \frac{v-u}{4}\left\{\int_{0}^{1}\left[\left(u^{\alpha-1} \zeta+(1-\zeta)\left(\frac{3 u+v}{4}\right)^{\alpha-1}\right)\left(u \zeta+(1-\zeta) \frac{3 u+v}{4}\right)-\left(\frac{3 u+v}{4}\right)^{\alpha}\right]\right. \\
& \times\left|h^{\prime}\left(u \zeta+(1-\zeta) \frac{3 u+v}{4}\right)\right| d \zeta \\
& +\int_{0}^{1}\left[\left(\left(\frac{3 u+v}{4}\right)^{\alpha-1} \zeta+(1-\zeta)\left(\frac{u+v}{2}\right)^{\alpha-1}\right)\left(\frac{3 u+v}{4} \zeta+(1-\zeta) \frac{u+v}{2}\right)-\left(\frac{3 u+v}{4}\right)^{\alpha}\right] \\
& \times\left|h^{\prime}\left(\frac{3 u+v}{4} \zeta+(1-\zeta) \frac{u+v}{2}\right)\right| d \zeta \\
& +\int_{0}^{1}\left[\left(\left(\frac{u+v}{2}\right)^{\alpha-1} \zeta+(1-\zeta)\left(\frac{u+3 v}{4}\right)^{\alpha-1}\right)\left(\frac{u+v}{2} \zeta+(1-\zeta) \frac{u+3 v}{4}\right)-\left(\frac{u+3 v}{4}\right)^{\alpha}\right] \\
& \times\left|h^{\prime}\left(\frac{u+v}{2} \zeta+(1-\zeta) \frac{u+3 v}{4}\right)\right| d \zeta \\
& +\int_{0}^{1}\left[\left(\left(\frac{u+3 v}{4}\right)^{\alpha-1} \zeta+(1-\zeta) v^{\alpha-1}\right)\left(\frac{u+3 v}{4} \zeta+(1-\zeta) v\right)-\left(\frac{u+3 v}{4}\right)^{\alpha}\right] \\
& \left.\times\left|h^{\prime}\left(\frac{u+3 v}{4} \zeta+(1-\zeta) v\right)\right| d \zeta\right\} .
\end{aligned}
$$


Since $\left|h^{\prime}\right|$ is convex on $[u, v]$ for any $\zeta \in[0,1]$, so (15) becomes

$$
\begin{aligned}
& \left|\Phi_{\alpha}(u, v)\right| \leq \frac{v-u}{4}\left\{\int_{0}^{1}\left[\left(u^{\alpha-1} \zeta+(1-\zeta)\left(\frac{3 u+v}{4}\right)^{\alpha-1}\right)\left(u \zeta+(1-\zeta) \frac{3 u+v}{4}\right)-\left(\frac{3 u+v}{4}\right)^{\alpha}\right]\right. \\
& \times\left[\zeta\left|h^{\prime}(u)\right|+(1-\zeta)\left|h^{\prime}\left(\frac{3 u+v}{4}\right)\right|\right] d \zeta+\int_{0}^{1}\left[\left(\left(\frac{3 u+v}{4}\right)^{\alpha-1} \zeta+(1-\zeta)\left(\frac{u+v}{2}\right)^{\alpha-1}\right)\right. \\
& \left.\times\left(\frac{3 u+v}{4} \zeta+(1-\zeta) \frac{u+v}{2}\right)-\left(\frac{3 u+v}{4}\right)^{\alpha}\right]\left[\zeta\left|h^{\prime}\left(\frac{3 u+v}{4}\right)\right|+(1-\zeta)\left|h^{\prime}\left(\frac{u+v}{2}\right)\right|\right] d \zeta \\
& +\int_{0}^{1}\left[\left(\left(\frac{u+v}{2}\right)^{\alpha-1} \zeta+(1-\zeta)\left(\frac{u+3 v}{4}\right)^{\alpha-1}\right)\left(\frac{u+v}{2} \zeta+(1-\zeta) \frac{u+3 v}{4}\right)-\left(\frac{u+3 v}{4}\right)^{\alpha}\right] \\
& \times\left[\zeta\left|h^{\prime}\left(\frac{u+v}{2}\right)\right|+(1-\zeta)\left|h^{\prime}\left(\frac{u+3 v}{4}\right)\right|\right] d \zeta+\int_{0}^{1}\left[\left(\left(\frac{u+3 v}{4}\right)^{\alpha-1} \zeta+(1-\zeta) v^{\alpha-1}\right)^{\alpha}\right. \\
& \left.\left.\times\left(\frac{u+3 v}{4} \zeta+(1-\zeta) v\right)-\left(\frac{u+3 v}{4}\right)^{\alpha}\right]\left[\zeta\left|h^{\prime}\left(\frac{u+3 v}{4}\right)\right|+(1-\zeta)\left|h^{\prime}(v)\right|\right] d \zeta\right\} .
\end{aligned}
$$

Simple calculation gives

$$
\begin{aligned}
& \left|\Phi_{\alpha}(u, v)\right| \leq \frac{v-u}{4}\left\{\left(\frac{1}{12}\left[4 u^{\alpha}+\left(\frac{3 u+v}{4}\right) u^{\alpha-1}+\left(\frac{3 u+v}{4}\right)^{\alpha-1} u-5\left(\frac{3 u+v}{4}\right)^{\alpha}\right]\right)\left|h^{\prime}(u)\right|\right. \\
& +\left(\frac{1}{12}\left[3 v^{\alpha}+\left(\frac{3 u+v}{4}\right) v^{\alpha-1}+\left(\frac{3 u+v}{4}\right)^{\alpha-1} v-5\left(\frac{3 u+v}{4}\right)^{\alpha}\right]\right)\left|h^{\prime}(v)\right| \\
& +\left(\frac { 1 } { 1 2 } \left[u^{\alpha}+\left(\frac{3 u+v}{4}\right) u^{\alpha-1}+\left(\frac{3 u+v}{4}\right)^{\alpha-1} u+\left(\frac{u+v}{2}\right)\left(\frac{3 u+v}{4}\right)^{\alpha-1}\right.\right. \\
& \left.\left.+\left(\frac{u+v}{2}\right)^{\alpha-1}\left(\frac{3 u+v}{4}\right)+\left(\frac{u+v}{2}\right)^{\alpha}-5\left(\frac{3 u+v}{4}\right)^{\alpha}\right]\right)\left|h^{\prime}\left(\frac{3 u+v}{4}\right)\right| \\
& +\left(\frac { 1 } { 1 2 } \left[7\left(\frac{u+v}{2}\right)^{\alpha}+\left(\frac{u+v}{2}\right)\left(\frac{3 u+v}{4}\right)^{\alpha-1}+\left(\frac{u+v}{2}\right)^{\alpha-1}\left(\frac{3 u+v}{4}\right)\right.\right. \\
& \left.\left.+\left(\frac{u+v}{2}\right)\left(\frac{u+3 v}{4}\right)^{\alpha-1}+\left(\frac{u+v}{2}\right)^{\alpha-1}\left(\frac{u+3 v}{4}\right)-5\left(\frac{3 u+v}{4}\right)^{\alpha}-5\left(\frac{u+3 v}{4}\right)^{\alpha}\right]\right)\left|h^{\prime}\left(\frac{u+v}{2}\right)\right| \\
& +\left(\frac { 1 } { 1 2 } \left[v^{\alpha}+\left(\frac{u+v}{2}\right)^{\alpha}+\left(\frac{u+3 v}{4}\right)\left(\frac{u+v}{2}\right)^{\alpha-1}+\left(\frac{u+3 v}{4}\right)^{\alpha-1}\left(\frac{u+v}{2}\right)\right.\right. \\
& \left.\left.\left.+v\left(\frac{u+3 v}{4}\right)^{\alpha-1}+v^{\alpha-1}\left(\frac{u+3 v}{4}\right)-5\left(\frac{u+3 v}{4}\right)^{\alpha}\right]\right)\left|h^{\prime}\left(\frac{u+3 v}{4}\right)\right|\right\} .
\end{aligned}
$$

This completes the proof of Theorem 2.

Corollary 1. With the similar assumptions of Theorem 2, if $\alpha=1$, then

$$
\begin{aligned}
\left|\Phi_{1}(u, v)\right| & \leq \frac{v-u}{4}\left[\frac{2 u-v}{12}\left|h^{\prime}(u)\right|+\frac{v-u}{12}\left|h^{\prime}(v)\right|\right. \\
& \left.+\frac{3 u+v}{48}\left|h^{\prime}\left(\frac{3 u+v}{4}\right)\right|+\frac{u+v}{24}\left|h^{\prime}\left(\frac{u+v}{2}\right)\right|+\frac{u+3 v}{48}\left|h^{\prime}\left(\frac{u+3 v}{4}\right)\right|\right] .
\end{aligned}
$$


Theorem 3. Let $h:[u, v] \subseteq \mathbb{R} \rightarrow \mathbb{R}$ be an $\alpha$-fractional differentiable mapping on $(u, v)$ with $0 \leq u<v$. If $D_{\alpha}(h) \in L_{\alpha}^{1}([u, v])$ and $\left|h^{\prime}\right|^{q}$ is convex on $[u, v]$, then the following inequality for conformable fractional integral holds:

$$
\begin{aligned}
\left|\Phi_{\alpha}(u, v)\right| & \leq \frac{v-u}{4}\left[\left(A_{1}(\alpha)\right)^{1-\frac{1}{q}}\left\{A_{2}(\alpha)\left|h^{\prime}(u)\right|^{q}+A_{3}(\alpha)\left|h^{\prime}\left(\frac{3 u+v}{4}\right)\right|^{q}\right\}^{\frac{1}{q}}\right. \\
& +\left(B_{1}(\alpha)\right)^{1-\frac{1}{q}}\left\{B_{2}(\alpha)\left|h^{\prime}\left(\frac{3 u+v}{4}\right)\right|^{q}+B_{3}(\alpha)\left|h^{\prime}\left(\frac{u+v}{2}\right)\right|^{q}\right\}^{\frac{1}{q}} \\
& +\left(C_{1}(\alpha)\right)^{1-\frac{1}{q}}\left\{C_{2}(\alpha)\left|h^{\prime}\left(\frac{u+v}{2}\right)\right|^{q}+C_{3}(\alpha)\left|h^{\prime}\left(\frac{u+3 v}{4}\right)\right|^{q}\right\}^{\frac{1}{q}} \\
& \left.+\left(D_{1}(\alpha)\right)^{1-\frac{1}{q}}\left\{D_{2}(\alpha)\left|h^{\prime}\left(\frac{u+3 v}{4}\right)\right|^{q}+D_{3}(\alpha)\left|h^{\prime}(v)\right|^{q}\right\}^{\frac{1}{q}}\right],
\end{aligned}
$$

where

$$
\begin{aligned}
A_{1}(\alpha)= & \frac{4 u(3 u+v)^{\alpha}-(4 u)^{\alpha+1}}{4^{\alpha}(v-u)(\alpha+1)}-\frac{\alpha(3 u+v)^{\alpha}}{4^{\alpha}}, \\
A_{2}(\alpha)= & \frac{(3 u+v)^{\alpha+2}-(4 u)^{\alpha+1}[\alpha(v-u)+(u+v)]}{4^{\alpha}(v-u)^{2}(\alpha+1)(\alpha+2)}-\frac{(3 u+v)^{\alpha}}{2^{2 \alpha+1}}, \\
A_{3}(\alpha)= & \frac{(4 u)^{\alpha+2}-(3 u+v)^{\alpha+2}(5 u-v)-\alpha(3 u+v)^{\alpha}(3 u-v)(u+v)}{4^{\alpha}(v-u)^{2}(\alpha+1)(\alpha+2)}-\frac{(3 u+v)^{\alpha}}{2^{2 \alpha+1}}, \\
B_{1}(\alpha)= & \frac{(2(u+v))^{\alpha+2}-(3 u+v)^{\alpha+1}}{4^{\alpha}(v-u)(\alpha+1)}-\frac{(3 u+v)^{\alpha}}{4^{\alpha}}, \\
B_{2}(\alpha)= & \frac{(2(u+v))^{\alpha+2}-(3 u+v)^{\alpha+1}(u+3 v)-\alpha(3 u+v)^{\alpha+1}(v-u)}{4^{\alpha}(v-u)^{2}(\alpha+1)(\alpha+2)}-\frac{(3 u+v)^{\alpha}}{2^{2 \alpha+1}} \\
B_{3}(\alpha)= & \frac{(3 u+v)^{\alpha+2}+4(2(u+v))^{\alpha+1}+\alpha(2(u+v))^{\alpha+1}(v-u)}{4^{\alpha}(v-u)^{2}(\alpha+1)(\alpha+2)}-\frac{(3 u+v)^{\alpha}}{2^{2 \alpha+1}}, \\
C_{1}(\alpha)= & \frac{4(u+3 v)^{\alpha+1}-(2(u+v))^{\alpha+2}}{4^{\alpha}(v-u)(\alpha+1)}-\frac{(u+3 v)^{\alpha}}{4^{\alpha}}, \\
C_{2}(\alpha)= & \frac{\alpha^{2}(v-u)^{2}(u+3 v)^{\alpha}+16 b(u+v)(u+3 v)^{\alpha}-3 \alpha\left(u^{2}+v^{2}\right)(u+3 v)^{\alpha}}{2^{2 \alpha+1}(v-u)^{2}(\alpha+1)(\alpha+2)} \\
& -\frac{8 b(2(u+v))^{\alpha+1}+2 \alpha(v-u)(2(u+v))^{\alpha+1}}{2^{2 \alpha+1}(v-u)^{2}(\alpha+1)(\alpha+2)}-\frac{(u+3 v)^{\alpha}}{2^{2 \alpha+1}}, \\
C_{3}(\alpha)= & \frac{(2(u+v))^{\alpha+2}+(u+3 v)^{\alpha+2}+\alpha(u+3 v)^{\alpha+2}-5(u+v)(u+3 v)^{\alpha+1}}{4^{\alpha}(v-u)^{2}(\alpha+1)(\alpha+2)},
\end{aligned}
$$




$$
\begin{aligned}
& D_{1}(\alpha)=\frac{(4 v)^{\alpha+1}-(u+3 v)^{\alpha+1}}{4^{\alpha}(\alpha+1)(v-u)}-\frac{(u+3 v)^{\alpha}}{2^{2 \alpha+1}} \\
& D_{2}(\alpha)=\frac{(4 v)^{\alpha+2}+(u+3 v)^{\alpha+1}(a-5 v)+\alpha(u+3 v)^{\alpha+1(v-u)}}{4^{\alpha}(v-u)^{2}(\alpha+1)(\alpha+2)} \\
& D_{3}(\alpha)=\frac{\alpha(4 v)^{\alpha+2}(v-u)-2(4 v)^{\alpha+1}(u+v)+(u+3 v)^{\alpha+2}}{4^{\alpha}(v-u)^{2}(\alpha+1)(\alpha+2)} .
\end{aligned}
$$

Proof. Using Lemma 1 and the property (4), we have

$$
\begin{aligned}
& \left|\Phi_{\alpha}(u, v)\right| \leq \frac{v-u}{4}\left[\int_{0}^{1}\left[\left(u \zeta+(1-\zeta) \frac{3 u+v}{4}\right)^{\alpha}-\left(\frac{3 u+v}{4}\right)^{\alpha}\right]\left|h^{\prime}\left(u \zeta+(1-\zeta) \frac{3 u+v}{4}\right)\right| d \zeta\right. \\
& +\int_{0}^{1}\left[\left(\frac{3 u+v}{4} \zeta+(1-\zeta) \frac{u+v}{2}\right)^{\alpha}-\left(\frac{3 u+v}{4}\right)^{\alpha}\right]\left|h^{\prime}\left(\frac{3 u+v}{4} \zeta+(1-\zeta) \frac{u+v}{2}\right)\right| d \zeta \\
& +\int_{0}^{1}\left[\left(\frac{u+v}{2} \zeta+(1-\zeta) \frac{u+3 v}{4}\right)^{\alpha}-\left(\frac{u+3 v}{4}\right)^{\alpha}\right]\left|h^{\prime}\left(\frac{u+v}{2} \zeta+(1-\zeta) \frac{u+3 v}{4}\right)\right| d \zeta \\
& +\int_{0}^{1}\left[\left(\frac{u+3 v}{4} \zeta+(1-\zeta) v\right)^{\alpha}-\left(\frac{u+3 v}{4}\right)^{\alpha}\right]\left|h^{\prime}\left(\frac{u+3 v}{4} \zeta+(1-\zeta) v\right)\right| d \zeta \\
& :=\frac{v-u}{4}\left(J_{1}+J_{2}+J_{3}+J_{4}\right) .
\end{aligned}
$$

It follows from the power-mean inequality that

$$
\begin{aligned}
J_{1} & \leq\left(\int_{0}^{1}\left[\left(u \zeta+(1-\zeta) \frac{3 u+v}{4}\right)^{\alpha}-\left(\frac{3 u+v}{4}\right)^{\alpha}\right] d \zeta\right)^{1-\frac{1}{q}} \\
& \times\left(\int_{0}^{1}\left[\left(u \zeta+(1-\zeta) \frac{3 u+v}{4}\right)^{\alpha}-\left(\frac{3 u+v}{4}\right)^{\alpha}\right]\left|h^{\prime}\left(u \zeta+(1-\zeta) \frac{3 u+v}{4}\right)\right|^{q} d \zeta\right)^{\frac{1}{q}} .
\end{aligned}
$$

Since $\left|h^{\prime}\right|^{q}$ is convex on $[u, v]$ for any $\zeta \in[0,1]$, we obtain

$$
\begin{aligned}
J_{1} & \leq\left(\int_{0}^{1}\left[\left(u \zeta+(1-\zeta) \frac{3 u+v}{4}\right)^{\alpha}-\left(\frac{3 u+v}{4}\right)^{\alpha}\right] d \zeta\right)^{1-\frac{1}{q}} \\
& \times\left(\left|h^{\prime}(u)\right|^{q} \int_{0}^{1}\left[\left(u \zeta+(1-\zeta) \frac{3 u+v}{4}\right)^{\alpha}-\left(\frac{3 u+v}{4}\right)^{\alpha}\right] \zeta d \zeta\right. \\
& \left.+\left|h^{\prime}\left(\frac{3 u+v}{4}\right)\right|^{q} \int_{0}^{1}\left[\left(u \zeta+(1-\zeta) \frac{3 u+v}{4}\right)^{\alpha}-\left(\frac{3 u+v}{4}\right)^{\alpha}\right](1-\zeta) d \zeta\right)^{\frac{1}{q}} \\
& =\left(A_{1}(\alpha)\right)^{1-\frac{1}{q}}\left\{A_{2}(\alpha)\left|h^{\prime}(u)\right|^{q}+A_{3}(\alpha)\left|h^{\prime}\left(\frac{3 u+v}{4}\right)\right|^{q}\right\}^{\frac{1}{q}}
\end{aligned}
$$

where we have used the facts that

$$
A_{1}(\alpha):=\int_{0}^{1}\left[\left(u \zeta+(1-\zeta) \frac{3 u+v}{4}\right)^{\alpha}-\left(\frac{3 u+v}{4}\right)^{\alpha}\right] d \zeta=\frac{4 u(3 u+v)^{\alpha}-(4 u)^{\alpha+1}}{4^{\alpha}(v-u)(\alpha+1)}-\frac{\alpha(3 u+v)^{\alpha}}{4^{\alpha}},
$$




$$
\begin{aligned}
A_{2}(\alpha) & :=\int_{0}^{1}\left[\left(u \zeta+(1-\zeta) \frac{3 u+v}{4}\right)^{\alpha}-\left(\frac{3 u+v}{4}\right)^{\alpha}\right] \zeta d \zeta \\
& =\frac{(3 u+v)^{\alpha+2}-(4 u)^{\alpha+1}[\alpha(v-u)+(u+v)]}{4^{\alpha}(v-u)^{2}(\alpha+1)(\alpha+2)}-\frac{(3 u+v)^{\alpha}}{2^{2 \alpha+1}}
\end{aligned}
$$

and

$$
\begin{aligned}
A_{3}(\alpha) & :=\int_{0}^{1}\left[\left(u \zeta+(1-\zeta) \frac{3 u+v}{4}\right)^{\alpha}-\left(\frac{3 u+v}{4}\right)^{\alpha}\right](1-\zeta) d \zeta \\
& =\frac{(4 u)^{\alpha+2}-(3 u+v)^{\alpha+2}(5 u-v)-\alpha(3 u+v)^{\alpha}(3 u-v)(u+v)}{4^{\alpha}(v-u)^{2}(\alpha+1)(\alpha+2)}-\frac{(3 u+v)^{\alpha}}{2^{2 \alpha+1}} .
\end{aligned}
$$

Analogously

$$
\begin{aligned}
& J_{2} \leq\left(B_{1}(\alpha)\right)^{1-\frac{1}{q}}\left\{B_{2}(\alpha)\left|h^{\prime}\left(\frac{3 u+v}{4}\right)\right|^{q}+B_{3}(\alpha)\left|h^{\prime}\left(\frac{u+v}{2}\right)\right|^{q}\right\}^{\frac{1}{q}}, \\
& J_{3} \leq\left(C_{1}(\alpha)\right)^{1-\frac{1}{q}}\left\{C_{2}(\alpha)\left|h^{\prime}\left(\frac{u+v}{2}\right)\right|^{q}+C_{3}(\alpha)\left|h^{\prime}\left(\frac{u+3 v}{4}\right)\right|^{q}\right\}^{\frac{1}{q}},
\end{aligned}
$$

and

$$
J_{4} \leq\left(D_{1}(\alpha)\right)^{1-\frac{1}{q}}\left\{D_{2}(\alpha)\left|h^{\prime}\left(\frac{u+3 v}{4}\right)\right|^{q}+D_{3}(\alpha)\left|h^{\prime}(v)\right|^{q}\right\}^{\frac{1}{q}} .
$$

Using $J_{1}, J_{2}, J_{3}$, and $J_{4}$ in (18), we obtain the desired inequality (17). This completes the proof of Theorem 3.

Corollary 2. With the similar assumptions of Theorem 3 , if $\alpha=1$, then

$$
\begin{aligned}
\left|\Phi_{1}(u, v)\right| & \leq \frac{v-u}{4}\left[\left(\frac{u-v}{8}\right)^{1-\frac{1}{q}}\left\{\left(\frac{11 u+v}{24}\right)\left|h^{\prime}(u)\right|^{q}+\left(\frac{5 u+v}{12}\right)\left|h^{\prime}\left(\frac{3 u+v}{4}\right)\right|^{q}\right\}^{\frac{1}{q}}\right. \\
& +\left(\frac{v-u}{8}\right)^{1-\frac{1}{q}}\left\{\left(\frac{2 u+v}{6}\right)\left|h^{\prime}\left(\frac{3 u+v}{4}\right)\right|^{q}+\left(\frac{7 u+5 b}{24}\right)\left|h^{\prime}\left(\frac{u+v}{2}\right)\right|^{q}\right\}^{\frac{1}{q}} \\
& +\left(\frac{u-v}{8}\right)^{1-\frac{1}{q}}\left\{\left(\frac{u-v}{12}\right)\left|h^{\prime}\left(\frac{u+v}{2}\right)\right|^{q}+\left(\frac{u-v}{24}\right)\left|h^{\prime}\left(\frac{u+3 v}{4}\right)\right|^{q}\right\}^{\frac{1}{q}} \\
& \left.+\left(\frac{v-u}{8}\right)^{1-\frac{1}{q}}\left\{\left(\frac{u+5 b}{12}\right)\left|h^{\prime}\left(\frac{u+3 v}{4}\right)\right|^{q}+\left(\frac{u+11 b}{24}\right)\left|h^{\prime}(v)\right|^{q}\right\}^{\frac{1}{q}}\right] .
\end{aligned}
$$

Theorem 4. Let $h:[u, v] \subseteq \mathbb{R} \rightarrow \mathbb{R}$ be an $\alpha$-fractional differentiable mapping on $(u, v)$ with $0 \leq u<v$. If $D_{\alpha}(h) \in L_{\alpha}^{1}([u, v])$ and $\left|h^{\prime}\right|^{q}$ is concave on $[u, v]$, then the following inequality for conformable fractional integral holds:

$$
\begin{aligned}
\left|\Phi_{\alpha}(u, v)\right| & \leq \frac{v-u}{4}\left[A_{1}(\alpha) h^{\prime}\left(\frac{P_{1}(\alpha)}{A_{1}(\alpha)}\right)\right. \\
& \left.+B_{1}(\alpha) h^{\prime}\left(\frac{P_{2}(\alpha)}{B_{1}(\alpha)}\right)+C_{1}(\alpha) h^{\prime}\left(\frac{P_{3}(\alpha)}{C_{1}(\alpha)}\right)+D_{1}(\alpha) h^{\prime}\left(\frac{P_{4}(\alpha)}{D_{1}(\alpha)}\right)\right]
\end{aligned}
$$


where $A_{1}(\alpha), B_{1}(\alpha), C_{1}(\alpha)$, and $D_{1}(\alpha)$ are given in Theorem 3 and

$$
\begin{aligned}
& P_{1}(\alpha)=\frac{2^{1-\alpha}(3 u+v)^{\alpha+2}-2^{2 \alpha+5}-2^{2 \alpha+1}(7 u+6 v)(v-u)(3 u+v)^{\alpha}-\alpha(7 u+v)(v-u)(3 u+v)^{\alpha}}{2^{2 \alpha+3}(v-u)(\alpha+2)}, \\
& P_{2}(\alpha)=\frac{2(2(u+v))^{\alpha+2}-8(u+v)^{2}(3 u+v)^{\alpha}-\alpha(5 u+3 v)(v-u)(3 u+v)^{\alpha}}{2^{2 \alpha+3}(v-u)(\alpha+2)}, \\
& P_{3}(\alpha)=\frac{8(u+v)^{2}(u+3 v)^{\alpha}-4 \alpha(u+v)^{\alpha}(u+3 v)^{\alpha}-\alpha(u+3 v)^{\alpha+2}-2^{\alpha+3}(u+v)^{\alpha+2}}{2^{2 \alpha+1}(v-u)(\alpha+2)}, \\
& P_{4}(\alpha)=\frac{2^{2 \alpha-1} \alpha(u+3 v)+4^{\alpha}(u+3 v)^{3}+4(4 v)^{\alpha+2}-4^{\alpha+2} v^{2}(u+3 v)-4(u+3 v)^{\alpha+2}-2^{2 \alpha+3} \alpha v^{2}(u+3 v)}{4^{\alpha+2}(v-u)(\alpha+2)} .
\end{aligned}
$$

Proof. By using the power-mean inequality and the concavity of $\left|h^{\prime}\right|^{q}$ for any $\zeta \in[0,1]$, we have

$$
\left|h^{\prime}\left(u \zeta+(1-\zeta) \frac{3 u+v}{4}\right)\right|^{q} \geq\left(\zeta\left|h^{\prime}(u)\right|+(1-\zeta)\left|\left(\frac{3 u+v}{4}\right)\right|\right)^{q} .
$$

This implies that

$$
\left|h^{\prime}\left(u \zeta+(1-\zeta) \frac{3 u+v}{4}\right)\right| \geq \zeta\left|h^{\prime}(u)\right|+(1-\zeta)\left|\left(\frac{3 u+v}{4}\right)\right| .
$$

This means that $\left|h^{\prime}\right|$ is also concave. Using inequality (20) in (18) and then applying the Jensen's integral inequality, we get

$$
\begin{aligned}
J_{1} & \leq\left(\int_{0}^{1}\left[\left(u \zeta+(1-\zeta) \frac{3 u+v}{4}\right)^{\alpha}-\left(\frac{3 u+v}{4}\right)^{\alpha}\right] d \zeta\right) \\
& \times h^{\prime}\left(\frac{\int_{0}^{1}\left[\left(u \zeta+(1-\zeta) \frac{3 u+v}{4}\right)^{\alpha}-\left(\frac{3 u+v}{4}\right)^{\alpha}\right]\left(u \zeta+(1-\zeta)^{\frac{3 u+v}{4}}\right) d \zeta}{\int_{0}^{1}\left[\left(u \zeta+(1-\zeta)^{\frac{3 u+v}{4}}\right)^{\alpha}-\left(\frac{3 u+v}{4}\right)^{\alpha}\right] d \zeta}\right) \\
& =A_{1}(\alpha) h^{\prime}\left(\frac{P_{1}(\alpha)}{A_{1}(\alpha)}\right)
\end{aligned}
$$

where we have used the facts that

$$
\begin{aligned}
& \int_{0}^{1}\left[\left(u \zeta+(1-\zeta) \frac{3 u+v}{4}\right)^{\alpha}-\left(\frac{3 u+v}{4}\right)^{\alpha}\right] d \zeta=A_{1}(\alpha)=\frac{4 u(3 u+v)^{\alpha}-(4 u)^{\alpha+1}}{4^{\alpha}(v-u)(\alpha+1)}-\frac{\alpha(3 u+v)^{\alpha}}{4^{\alpha}}, \\
& \int_{0}^{1}\left[\left(u \zeta+(1-\zeta) \frac{3 u+v}{4}\right)^{\alpha}-\left(\frac{3 u+v}{4}\right)^{\alpha}\right]\left(u \zeta+(1-\zeta) \frac{3 u+v}{4}\right) d \zeta=P_{1}(\alpha) \\
& =\frac{2^{1-\alpha}(3 u+v)^{\alpha+2}-2^{2 \alpha+5}-2^{2 \alpha+1}(7 u+6 v)(v-u)(3 u+v)^{\alpha}-\alpha(7 u+v)(v-u)(3 u+v)^{\alpha}}{2^{2 \alpha+3}(v-u)(\alpha+2)} .
\end{aligned}
$$

Similarly, we get $J_{2} \leq B_{1}(\alpha) h^{\prime}\left(\frac{P_{2}(\alpha)}{B_{1}(\alpha)}\right), J_{3} \leq C_{1}(\alpha) h^{\prime}\left(\frac{P_{3}(\alpha)}{C_{1}(\alpha)}\right)$ and $J_{4} \leq D_{1}(\alpha) h^{\prime}\left(\frac{P_{4}(\alpha)}{D_{1}(\alpha)}\right)$.

Using $J_{1}, J_{2}, J_{3}$, and $J_{4}$ in (18), we obtain the required inequality (19). This completes the proof of Theorem 4.

Corollary 3. With the similar assumptions of Theorem 4 , if $\alpha=1$, then

$$
\left|\Phi_{1}(u, v)\right| \leq \frac{v-u}{4}\left[A_{1}(\alpha) h^{\prime}\left(\frac{P_{1}(\alpha)}{A_{1}(\alpha)}\right)+B_{1}(\alpha) h^{\prime}\left(\frac{P_{2}(\alpha)}{B_{1}(\alpha)}\right)+C_{1}(\alpha) h^{\prime}\left(\frac{P_{3}(\alpha)}{C_{1}(\alpha)}\right)+D_{1}(\alpha) h^{\prime}\left(\frac{P_{4}(\alpha)}{D_{1}(\alpha)}\right)\right],
$$


where

$$
A_{1}(1)=\frac{u-v}{8}, \quad B_{1}(1)=\frac{v-u}{8}, \quad D_{1}(1)=\frac{u-v}{8}, \quad D_{1}(1)=\frac{v-u}{8}
$$

and

$$
\begin{array}{ll}
P_{1}(1)=\frac{(u-v)(11 u+v)}{96}, & P_{2}(1)=\frac{(v-u)(7 u+5 v)}{96}, \\
P_{3}(1)=\frac{(u-v)(5 u+7 v)}{96}, & P_{4}(1)=\frac{(v-u)(u+11 v)}{96} .
\end{array}
$$

\section{Conclusions}

In this work, we have established new conformable fractional integral inequalities of Hermite-Hadamard type for convex functions. As a special case, if we substitute $\alpha=1$ into the general definition of conformable fractional integrals (Definition 2), we obtain the classical integrals. In view of this, we obtained some new inequalities of Hermite-Hadamard type for convex functions involving classical integrals.

Author Contributions: The authors contributed equally to this work. All authors read and approved the final manuscript.

Funding: This research received no external funding.

Acknowledgments: The authors are thankful to the anonymous referees for their careful corrections to and valuable comments on the original version of this paper.

Conflicts of Interest: The authors declare no conflict of interest.

\section{References}

1. Gavrea, B.; Gavrea, I. On some Ostrowski type inequalities. Gen. Math. 2010, 18, 33-44.

2. Ciatti, P.; Cowling, M.G.; Ricci, F. Hardy and uncertainty inequalities on stratified Lie groups. Adv. Math. 2015, $277,365-387$.

3. Gunawan, H.; Eridani, E. Fractional integrals and generalized Olsen inequalities. Kyungpook Math. J. 2009, 49, 31-39. [CrossRef]

4. Sawano, Y.; Wadade, H. On the Gagliardo-Nirenberg type inequality in the critical Sobolev-orrey space. J. Fourier Anal. Appl. 2013, 19, 20-47. [CrossRef]

5. $\mathrm{Wu}, \mathrm{S}$.-H. On the weighted generalization of the Hermite-Hadamard inequality and its applications. Rocky Mt. J. Math. 2009, 39, 1741-1749. [CrossRef]

6. Mohammed, P.O.; Sarikaya, M.Z. Hermite-Hadamard type inequalities for $F$-convex function involving fractional integrals. J. Inequalities Appl. 2018, 2018, 359. [CrossRef]

7. Bakula, M.K.; Ozdemir, M.E.; Pečarić, J. Hadamard type inequalities for $m$-convex and $(\alpha, m)$-convex functions. J. Inequal. Pure Appl. Math. 2008, 9, 96.

8. Alomari, M.; Darus, M.; Kirmaci, U.S. Refinements of Hadamard-type inequalities for quasi-convex functions with applications to trapezoidal formula and to special means. Comp. Math. Appl. 2010, 59, 225-232. [CrossRef]

9. Yin, H.-P.; Qi, F. Hermite-Hadamard type inequalities for the product of $(\alpha, m)$-convex functions. J. Nonlinear Sci. Appl. 2015, 8, 231-236. [CrossRef]

10. Noor, M.A.; Noor, K.I.; Awan, M.U. Some quantum estimates for Hermite-Hadamard inequalities. Appl. Math. Comput. 2015, 251, 675-679. [CrossRef]

11. Kilbas, A.A.; Srivastava, H.M.; Trujillo, J.J. Theory and Applications of Fractional Differential Equations; Elsevier B.V.: Amsterdam, The Netherlands, 2006. 
12. Shi, D.-P.; Xi, B.-Y.; Qi, F. Hermite-Hadamard type inequalities for Riemann-Liouville fractional integrals of $(\alpha, m)$-convex functions. Fract. Differ. Calc. 2014, 4, 33-45. [CrossRef]

13. Mohammed, P.O. Inequalities of type Hermite-Hadamard for fractional integrals via differentiable convex functions. Turk. J. Anal. Number Theory 2016, 4, 135-139.

14. Hammad, M.A.; Khalil, R. Conformable fractional heat differential equations. Int. J. Pure Appl. Math. 2014, 94, 215-221. [CrossRef]

15. Hammad, M.A.; Khalil, R. Abel's formula and wronskian for conformable fractional differential equations. Int. J. Differ. Equations Appl. 2014, 13, 177-183.

16. Khalil, R.; Horani, M.A.; Yousef, A.; Sababheh, M. A new definition of fractional derivative. J. Comput. Appl. Math. 2014, 264, 65-70. [CrossRef]

17. Abdeljawad, T. On conformable fractional calculus. J. Comput. Appl. Math. 2015, 279, 57-66. [CrossRef]

18. Atangana, A.; Baleanu, D.; Alsaedi, A. New properties of conformable derivative. Open Math. 2015, 13, 889-898. [CrossRef]

19. Iyiola, O.S.; Nwaeze, E.R. Some new results on the new conformable fractional calculus with application using D'Alambert approach. Progr. Fract. Differ. Appl. 2016, 2, 115-122. [CrossRef]

20. Katugampola, U. A new fractional derivative with classical properties. arXiv 2014, arXiv:1410.6535v2.

21. Zhao, D.; Luo, M. General conformable fractional derivative and its physical interpretation. Calcolo 2017, 54, 903-917. [CrossRef]

(C) 2019 by the authors. Licensee MDPI, Basel, Switzerland. This article is an open access article distributed under the terms and conditions of the Creative Commons Attribution (CC BY) license (http:/ / creativecommons.org/licenses/by/4.0/). 